\title{
Evaluation of the basic erosive wear examination (BEWE) for use in general dental practice
}

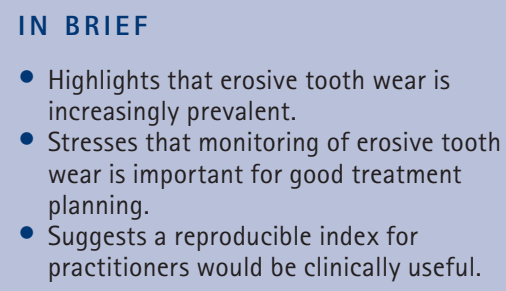

\author{
B. Dixon, ${ }^{1}$ M. O. Sharif, ${ }^{2}$ F. Ahmed, ${ }_{1}^{3}$ A. B. Smith, ${ }_{1}^{4}$ D. Seymour ${ }^{5}$ \\ and P. A. Brunton ${ }^{6}$
}

- Highlights that erosive tooth wear is

esingly prevalent.

wear is important for good treatment

lanning.

practitioners would be clinically useful.

Objective The basic erosive wear examination (BEWE) is a relatively new index proposed for the screening and recording of tooth wear in adults. The aim of this study was to test the validity and reliability of the BEWE. Design Cross sectional study of a sample of 164 adult patients. Setting General dental practice in East Lancashire, UK in 2010. Subjects Patients attending for routine examination or treatment. Main outcome measures By screening patients with the BEWE and comparing the results to the established tooth wear index (TWI) the sensitivity and specificity of the BEWE was established. Results The BEWE predicted moderate to severe wear (BEWE grade 3 ) with a sensitivity of $48.6 \%$ and a specificity of $96.1 \%$, and predicted severe wear with a sensitivity of $90.9 \%$ and a specificity of $91.5 \%$ (also BEWE score 3 ). Inter- and intra-examiner reliability for the BEWE were both moderate ( $\kappa_{w}=0.43$ and 0.57 respectively). Conclusion(s) BEWE scores show a similar distribution to TWI scores and the examination is an effective screening test for severe tooth wear. The moderate levels of examiner reliability suggests the BEWE scores should be interpreted with some caution.

\section{INTRODUCTION}

Tooth wear is a growing problem. ${ }^{1}$ Four to five percent of 15-year-olds and $11 \%$ of adults in the UK have been shown to suffer from tooth wear; ${ }^{2,3}$ these figures demonstrate an increase over a 10-year period. ${ }^{4}$ The consequences of tooth wear on quality of life varies from pain and discomfort to a reduction in satisfaction in appearance with a potential disruption to eating capacity. ${ }^{5}$

Tooth wear has been shown to be multi-factorial in origin and often more than one form of tooth wear will be present in any one case. Erosion is one of the commonest causes of tooth wear; it has been defined as the loss of tooth substance resulting from chemical action, not involving bacteria. ${ }^{6-11}$

General Dental Practitioner, Leeds Dental Institute, University of Leeds, Leeds; ${ }^{2}$ NIHR Academic Clinical Fellow - Orthodontics, ${ }^{3}$ Dental Foundation Year Two Trainee, School of Dentistry, University of Manchester, Manchester: ${ }^{4}$ Lecturer in Quantitative Methods, ${ }^{5}$ Speciality Registrar in Restorative Dentistry, ${ }^{6 *}$ Professor of Restorative Dentistry, Leeds Dental Institute, University of Leeds, Leeds

${ }^{*}$ Corresponding to: Professor Paul A. Brunton Email: p.a.brunton@leeds.ac.uk

\section{Online article number E4}

Refereed Paper - accepted 14 April 2012

DOI: 10.1038/sj.bdj.2012.670

${ }^{\circ}$ British Dental Journal 2012; 213: E4
The management of tooth wear needs to be preceded by an accurate diagnosis and documentation of its severity. Despite a number of proposed indices for recording tooth wear being in existence, ${ }^{12-14}$ currently there is no universally accepted method for recording erosive tooth wear in general dental practice. The most popular index for recording tooth wear, the tooth wear index (TWI) was proposed in 1984 by Smith and Knight. It was designed for epidemiological studies, not the clinical treatment of individual patients, and it continues to enjoy wide use in epidemiological studies of tooth wear. ${ }^{15}$ The TWI consists of a five point scale in which the cervical, buccal/ labial, incisal/occlusal and lingual/palatal surfaces are evaluated. Identifying exposed dentine is the main distinguishing factor of the five point scale. One problem with this is that the ability to visually identify exposed dentine has been shown to be inconsistent from examiner to examiner. ${ }^{16}$ Other barriers to its implementation in practice are that it is time consuming and the evaluation is restricted in that heavily restored surfaces are excluded from the TWI score and so the score may not be a reflection of the clinical picture.

The basic erosive wear examination (BEWE) was described in 2008 by Bartlett et al. ${ }^{17,18}$ and was developed to provide a simpler way to monitor and record the severity and progress of erosive tooth wear in general practice. It is a partial scoring system similar to the basic periodontal examination (BPE), providing an efficient process for screening and recording erosion on a four point scale with the level of wear on the worst affected tooth surface in each sextant noted only. Due to the similarity with the BPE conceptually, the general dental practitioner would only require to adapt their understanding of the BPE to be able to implement the BEWE. The BEWE also incorporates a 'risk score', which is calculated by adding together the sums of the scores for all sextants. The risk score has been suggested to provide an indication of the individual patient's level of risk of tooth wear and therefore a guide to their clinical management. The BEWE examination criteria and risk score grading are shown in Tables 1 and 2 .

To date there have been no studies investigating the reliability of the BEWE in comparison to the currently accepted and used index of evaluation (TWI). A previous study ${ }^{17}$ on the reliability of the BEWE compared it to the visual erosion dental examination, where only erosive lesions were evaluated. However, tooth wear is 
Table 1 Basic erosive wear examination criteria

\begin{tabular}{l|l}
\hline Score & Criteria \\
\hline 0 & No erosive tooth wear \\
\hline 1 & Initial loss of surface texture \\
\hline 2 & Distinct defect; hard tissue loss $<50 \%$ of the surface area \\
\hline 3 & Hard tissue loss $\geq 50 \%$ of the surface area \\
\hline
\end{tabular}

Table 2 Basic erosive wear examination risk score grading

\begin{tabular}{l|l}
\hline Risk level & Cumulative score of all sextants \\
\hline None & Less than or equal to 2 \\
\hline Low & Between 3 and 8 \\
\hline Medium & Between 9 and 13 \\
\hline High & 14 and over
\end{tabular}

multifactorial, ${ }^{9}$ therefore in this current study all forms of tooth wear were assessed with the BEWE. The primary aim of this study is to evaluate whether the BEWE is reliable for screening and recording tooth wear in adults and to compare its reliability to the TWI. Reliability was assessed through sensitivity, specificity and risk scores.

\section{METHODS AND MATERIALS}

This study included 164 adult patients attending for routine dental care following sample size calculations based on the expected prevalence of wear. The age varied between 18 and 82 years (mean age $42.8 \pm$ SD 13.9) and all were recruited from a single general dental practice in Rawtenstall, East Lancashire, from May to July 2010. Table 3 shows the distribution of age and gender of the patients. Patients excluded from the study were:

1. Patients attending for urgent or nonroutine treatment

2. Patients unable to provide informed consent

3. Patients without at least one tooth remaining in each sextant.

This study was reviewed and approved by the South Yorkshire Research Ethics Committee. R\&D approval was obtained from East Lancashire PCT. All patients were provided with a patient information sheet and were required to sign informed written consent forms before being enrolled into the study.

All teeth were dried with compressed air and examined without the use of magnification for tooth wear under normal dental surgery conditions. The most severely affected tooth in each sextant was identified and the BEWE and TWI scores recorded. For the TWI all tooth surfaces were also recorded. Missing teeth and heavily restored surfaces were excluded; however, the remaining surfaces were scored. Teeth with trauma and developmental defects were eliminated from the score.

To assess intra-examiner reliability, dental impressions were made and study casts fabricated for a randomly selected sub-sample of 29 participants. The casts were assessed and BEWE scores recorded. The assessment and scoring of the study casts was repeated again by the same examiner after three weeks $\left(\kappa_{\mathrm{w}}=0.57\right)$. Inter-examiner reliability was assessed using a randomly selected sub-sample of 30 participants. A second examiner, blind to the first examiner's score, recorded a second BEWE $\left(\kappa_{\mathrm{w}}=0.43\right)$.

Statistical analysis of the results was carried out using the SPSS Version 13.

In order to compare the four point score of the BEWE with the five point score of the TWI, the BEWE sextant score of 3 was tested both as a predictor of moderate to severe wear (against a TWI score of 3 or 4) and as a predictor of severe wear (against a TWI score of 4). Two sets of sensitivity/specificity calculations were therefore carried out.

In order to assess the value of the cumulative 'risk score' in predicting risk, a number of TWI measures were compared to the
Table 3 Age and gender distribution of study participants

\begin{tabular}{|l|l|l|} 
Age & $\begin{array}{l}\text { Frequency } \\
\text { male }\end{array}$ & $\begin{array}{l}\text { Frequency } \\
\text { female }\end{array}$ \\
\hline$\geq 15 \times<20$ & 2 & 2 \\
\hline$\geq 20 \times<25$ & 5 & 9 \\
\hline$\geq 25 \times<30$ & 10 & 13 \\
\hline$\geq 30 \times<35$ & 4 & 8 \\
\hline$\geq 35 \times<40$ & 10 & 11 \\
\hline$\geq 40 \times<45$ & 12 & 12 \\
\hline$\geq 45 \times<50$ & 10 & 8 \\
\hline$\geq 50 \times<55$ & 4 & 8 \\
\hline$\geq 55 \times<60$ & 9 & 7 \\
\hline$\geq 60 \times<65$ & 9 & 1 \\
\hline$\geq 65 \times<70$ & 2 & 4 \\
\hline$\geq 70 \times<75$ & 0 & 0 \\
\hline$\geq 75 \times<80$ & 2 & 0 \\
\hline$\geq 80 \times<85$ & 2 & 1 \\
\hline
\end{tabular}

cumulative BEWE score. The TWI measures selected were the proportion of surfaces scoring 2 (wear into dentine) or higher, scoring 3 (extensive dentine exposure) or higher and scoring 4 (exposure of pulp or secondary dentine). Correlation was tested with Pearson's correlation coefficient and a linear regression analysis.

\section{RESULTS}

A total of 984 sextants and a total of 15,125 tooth surfaces (on 4,212 teeth) were scored with the BEWE and TWI respectively. Patient characteristics are shown in Table 3.

Ninety-six percent of patients were deemed to have tooth wear as determined by the BEWE in comparison to the TWI which detected tooth wear in 98\% of patients. Severity of tooth wear according to each index is shown in Table 4.

of the 984 sextants examined, moderate to severe wear (score 3 or 4) was found to have a varied distribution with the two indices. The BEWE determined that $4.0 \%$ of sextants $(n=39)$ had moderate to severe wear (score 3) in comparison to the TWI which determined that $6.8 \%(n=67)$ of sextants had moderate to severe wear (score 3 or 4 ). The most common sextants with moderate to severe wear from the BEWE were the upper anterior $(\mathrm{n}=10)$ and upper right sextants $(\mathrm{n}=10)$, the TWI scoring showed that the upper 


\begin{tabular}{|c|c|c|c|c|c|c|c|c|}
\hline \multicolumn{2}{|c|}{$\begin{array}{l}\text { TWI score of highest } \\
\text { scoring surface }\end{array}$} & 0 (no wear) & $\begin{array}{l}1 \text { (enamel } \\
\text { wear) }\end{array}$ & $\begin{array}{l}2 \text { (wear into } \\
\text { dentine) }\end{array}$ & $\begin{array}{l}3 \text { (more exten- } \\
\text { sive } \\
\text { dentine } \\
\text { exposure) }\end{array}$ & $\begin{array}{l}4 \text { (exposure } \\
\text { of pulp or } \\
\text { secondary } \\
\text { dentine) }\end{array}$ & Total & Percentage \\
\hline \multirow{6}{*}{$\begin{array}{l}\text { BEWE score of } \\
\text { highest scoring } \\
\text { sextant }\end{array}$} & $\begin{array}{l}0 \text { (no erosive } \\
\text { teeth wear) }\end{array}$ & 4 & 2 & 0 & 0 & 0 & 6 & $3.7 \%$ \\
\hline & $\begin{array}{l}1 \text { (initial loss } \\
\text { of surface } \\
\text { texture) }\end{array}$ & 0 & 32 & 10 & 1 & 0 & 43 & $26.2 \%$ \\
\hline & $\begin{array}{l}2 \text { (distinct } \\
\text { defect; hard } \\
\text { tissue loss } \\
<50 \% \text { of the } \\
\text { surface area }\end{array}$ & 0 & 6 & 68 & 17 & 1 & 92 & $56.1 \%$ \\
\hline & $\begin{array}{l}3 \text { (hard tissue } \\
\text { loss } \geq 50 \text { of the } \\
\text { surface area) }\end{array}$ & 0 & 0 & 5 & 8 & 10 & 23 & $14.0 \%$ \\
\hline & Total & 4 & 40 & 83 & 26 & 11 & \multirow[t]{2}{*}{164} & \multirow[t]{2}{*}{$100 \%$} \\
\hline & Percentage & $2.4 \%$ & $24.4 \%$ & $50.6 \%$ & $15.9 \%$ & $6.7 \%$ & & \\
\hline
\end{tabular}

anteriors $(\mathrm{n}=23)$ and lower anterior sextants $(n=24)$ were the worst affected in the population evaluated.

A sensitivity of $48.6 \%$ and a specificity of $96.1 \%$ for a score of 3 (moderate to severe wear) in a sextant was recorded for the BEWE in relation to the TWI. The positive predictive value was $78.3 \%$ and the negative predictive value was $6.5 \%$. A sensitivity of $90.9 \%$ and a specificity of $91.5 \%$ for a score of 3 (severe) with the BEWE and score 4 (severe wear) with TWI in a sextant was recorded. The positive predictive value of $43.5 \%$ and the negative predictive value of $99.3 \%$ were found.

The BEWE risk score ranged from 0 to 16 (mean 6.3, \pm SD 3.72, median 6). The Pearson correlation coefficient was 0.80 and the equation of the regression line was that the proportion of surfaces with wear into dentine equalled $-0.032+(0.015 \times$ risk score $)$.

\section{DISCUSSION}

Tooth wear is an on-going process that occurs to some degree in all dentate individuals. Often it does not require any clinical intervention but where there are symptoms, aesthetic or functional problems, intervention may be necessary.

Accurate techniques for measuring tooth surface loss in the laboratory have yet to be successfully adapted to the clinical setting. Since the TWI is only one way of recording tooth wear and not a gold standard, the concept of sensitivity and specificity should be regarded with caution.
Where the BEWE shows the presence of tooth wear but the TWI does not, this is not a 'false positive' but only indicates a condition that is identified by one index but not the other. However, the TWI is widely used and accepted; by contrast the BEWE is novel and relatively untested. If its results map closely to the TWI, this will help to show its validity.

Overall the distribution of BEWE scores appeared similar to that of TWI scores. The presence or absence of moderate or severe wear for a BEWE score of 3 was found to be $85.4 \%$ accurate in comparison to the TWI. As for the BEWE score of 3 acting as an indicator of severe wear, it was found to be $91.5 \%$ accurate. The sensitivity and specificity of the BEWE for moderate to severe wear was $48.6 \%$ and $96.1 \%$ respectively, the sensitivity and specificity of the BEWE for severe wear were $90.9 \%$ and 91.5\% respectively.

The low sensitivity score for moderate to severe tooth wear of the BEWE indicates that the majority of patients with moderate to severe wear did not have a BEWE score of 3. However, the high specificity score shows that there are few false positives. The presence of a BEWE score of 3 in any sextant is not necessarily an appropriate test for the presence of moderate to severe wear relative to the TWI. As is to be expected, using a BEWE score of 3 as a predictor of the narrower diagnostic category of severe wear (TWI score 4), the sensitivity increased (from $48.6 \%$ to $90.9 \%$ ) but the specificity fell $(96.1 \%$ to $91.5 \%)$.
One possible explanation for the differing results is that the indices have a different number of categories in their grading system, with the BEWE having four and the TWI having five, making direct comparisons difficult. It should be noted that the BEWE uses tissue loss over $50 \%$ of the surface to distinguish between grades 2 and 3, unlike the TWI which makes the distinction at enamel loss over one third of the surface.

There was moderate agreement in interexaminer $\left(\kappa_{w}=0.43\right)$ and intra-examiner reliablility $\left(\kappa_{w}=0.57\right)$. These results should not be regarded as definitive as they represent the assessments of only two examiners (for the inter-examiner reliability score) and one examiner (for the intra-examiner reliability score) respectively. These results indicate that the BEWE is indeed assessing a measurable construct with some degree of repeatability, but some potential for variability exists in scores recorded by different examiners or at different times, and results obtained from scoring study casts may not be entirely representative of the clinical picture.

The risk score element of the BEWE is strongly correlated to the proportion of surfaces with exposed dentine but is subject to unacceptable variation between examiners. Based on the results of this study its use is therefore not recommended.

A literature review has revealed that at the time of writing this paper, that only one study has been published to assess the reliability of the BEWE index. In this 
study Mulic et al. used the BEWE and the visual erosion dental examination (VEDE) scoring system (a five-point scale similar to the Eccles index). ${ }^{19}$ Mulic et al. assessed inter- and intra-examiner reliability of the VEDE scoring system. Only lesions considered to be obviously erosive were recorded. No direct statistical comparison was made between the results recorded with the two systems, although the inter- and intra-examiner reliability were similar.

The BEWE is a simple screening tool which is designed for general dental practice. The BEWE alerts clinicians to the presence of severe tooth wear, a condition of increasing prevalence in the UK from which further management would stem. It is based on a four point grading system (0-3) to allow efficient analysis of patients with tooth wear. The BEWE negates the unreliable evaluation of exposed dentine in assessing tooth wear and can be used not only with patients but also to evaluate casts.

The results of this study suggest that the BEWE is an appropriate screening tool to identify the presence or absence of severe tooth wear in general dental practice. However, before using the BEWE as an index for carrying out populationbased studies with multiple examiners, some form of calibration exercise is to be recommended in order to ensure good agreement.

\section{CONCLUSION}

The BEWE appears to be an appropriate tool for the measuring and recording of tooth wear of multi-factorial aetiology in a general dental practice setting, although a degree of caution needs to be applied when comparing results between clinicians or over time. The examination can also be used on study casts provided that the difficulty of assessing the condition of enamel from a study cast is recognised.

1. Van't Spijker A, Rodriguez J M, Kreulen C M, Bronkhorst E M, Bartlett D W, Creugers N H. Prevalence of tooth wear in adults. Int $J$ Prosthodont 2009; 22: 35-42.

2. Chadwick B, Pendry L. Non carious dental condition Children's dental health in the United Kingdom 2003. London: The Stationary Office, 2004.

3. Nunn J, Morris J, Pine C, Pitts N B, Bradnock G, Steele J. The condition of teeth in the UK in 1998 and implications for the future Br Dent J2000: 189: 639-644.

4. O'Brien M. Non carious dental conditions. Children's dental health in the United Kingdom 1993. London: The Stationary Office, 1994.

5. Al-Omiri M K, Lamey P J, Clifford T. Impact of tooth wear on daily living. Int J Prosthodont 2006: 19: 601-605.

6. Deery C, Wagner M L, Longbottom C, Simon R
Nugent Z J. The prevalence of dental erosion in a United States and a United Kingdom sample of adolescents. Pediatr Dent2000; 22: 505-510.

7. Grippo J O, Simring M, Schreiner S. Attrition, abrasion, corrosion and abfraction revisited: a new perspective on tooth surface lesions. J Am Dent Assoc 2004: 135: 1,109-1,118.

8. Seligman D A, Pullinger A G, Solberg W K. The prevalence of dental attrition and its association with factors of age, gender, occlusion, and TMJ symptomatology. J Dent Res 1988; 67(10): 1323-1333.

9. Lussi A. Dental erosion: clinical diagnosis and case history taking. Eur J Oral Sci 2002; 104: 191-198.

10. Zero D T. Etiology of dental erosion - extrinsic factors. Eur J Oral Sci 1996; 104: 162-177.

11. Scheutzel P. Etiology of dental erosion - intrinsic factors Eur J Oral Sci.1996; 104: 178-190.

12. Eccles J D, Jenkins W G. Dental erosion and diet. J Dent 1974; 2: 153-159.

13. Smith B G, Knight J K. An index for measuring the wear of teeth. Br Dent J 1984; 156: 435-438.

14. Lussi A, Schaffner M, Hotz P, Suter P. Dental erosion in a population of Swiss adults. Community Dent Oral Epidemiol 1991; 19: 286-290.

15. Bardsley P F. The evolution of tooth wear indices. Clin Oral Investig 2008; 12: S15-S19.

16. Ganss C, Klimek J, Lussi A. Accuracy and consistency of the visual diagnosis of exposed dentine on worn occlusal/incisal surfaces. Caries Res 2006; 40: 208-212.

17. Young A, Amaechi B T, Dugmore $C$ et al. Current erosion indices - flawed or valid? Summary. Clin Oral Investig 2008; 12:(Suppl 1) S59-S63.

18. Bartlett D, Ganss C, Lussi A. Basic erosive wear examination (BEWE): a new scoring system for scientific and clinical needs. Clin Oral Investig 2008; 12:(Suppl 1) S65-S68.

19. Mulic A, Tveit A B, Wang N J, Hove L H, Espelid I, Skaare A B. Reliability of two clinical scoring systems for dental erosive wear. Caries Res 2010; 44: 294-299. 